\title{
BMJ Open CUPID: a protocol of a randomised controlled trial to identify characteristics of similar Chinese patent medicines
}

\author{
Hongbo Cao, ${ }^{1,2}$ Jingbo Zhai, ${ }^{1}$ Nan Li, ${ }^{1}$ Hongxia Cao, ${ }^{3}$ Xiang Lei, ${ }^{2}$ Wei Mu, ${ }^{2}$ \\ Zhi Liu, ${ }^{1}$ Hui Wang, ${ }^{1}$ Hongcai Shang ${ }^{1}$
}

To cite: Cao H, Zhai J, Li N, et al. CUPID: a protocol of a randomised controlled trial to identify characteristics of similar Chinese patent medicines. BMJ Open 2014;4:e006753 doi:10.1136/bmjopen-2014006753

- Prepublication history for this paper is available online To view these files please visit the journal online (http://dx.doi.org/10.1136/ bmjopen-2014-006753)

Received 27 September 2014 Accepted 31 October 2014

\section{CrossMark}

${ }^{1}$ Tianjin Institute of Clinical Evaluation, Tianjin University of Traditional Chinese Medicine, Tianjin, China ${ }^{2}$ Center for Evidence-Based Medicine, Tianjin University of Traditional Chinese Medicine, Tianjin, China ${ }^{3}$ Department of Geriatrics, Gansu Hospital of Traditional Chinese Medicine, Lanzhou, China

\section{Correspondence to} Hongcai Shang; shanghongcai@foxmail.com and

Hongbo Cao;

caohongboebm@foxmail.com

\section{ABSTRACT}

Introduction: Traditional Chinese medicine (TCM) has accumulated some experience in curing stable angina pectoris (SAP) and efficacy has been demonstrated. Chinese patent medicines, known as modern dosage forms of TCM, can attain the desired effect in clinical application only with the guidance of TCM syndrome theory. However, due to their use by a large number of persons with little knowledge of TCM theories and practices, their efficacy and reputation have been seriously affected.

Method and analysis: Two common syndrome types of SAP in TCM, 'qi deficiency and blood stasis' and 'qi stagnation and blood stasis', will be studied in 144 subjects from four TCM hospitals in Tianjin in China using a partial crossover design. The two syndromes will be broken down into six symptom combinations; patients will select a combination of the most distressing to them, and then will be randomised into two groups. Each group, on the basis of routine medication, will be administered one kind of Chinese patent drug: Qishenyiqi Dripping Pills or Compound Danshen Dripping Pills. The treatment characteristics of the two medicines will be evaluated with the COMEPIO method developed by our research team.

Ethics and dissemination: This protocol has been approved by the medical ethics committee of Tianjin University of TCM (registration number TJUTCMEC20130005). The study is safe and reliable.

Trial registration number: Chinese clinical trials register ChiCTR-TTRCC-14004406.

\section{BACKGROUND}

Chinese patent medicines for stable angina pectoris

Stable angina pectoris (SAP) is a common coronary artery disease, its occurrence and mortality rate are on the rise. Though a number of methods are available for its control, it is still an area of high concern, especially for a series of relevant clinical symptoms associated with this disease. ${ }^{1}$ In China, traditional Chinese medicine (TCM) is a prevailing comparative and alternative medicine. ${ }^{2}{ }^{3}$ In the past few

\section{Strengths and limitations of this study}

- PI0+PRO: evaluation by combination of PIO and PRO. Patients select symptom type and evaluate the efficacy by themselves, with a popular 'patentcentred evaluation' in modern medicine.

- TCM syndrome+symptom (combinations): evaluation by combination of TCM syndrome and symptom (combinations). To find the efficacy of Chinese patent medicines by breaking down TCM syndromes into different symptoms or symptom combinations.

- The practicability and feasibility of the methodology in this study needs to be tested in practice.

years, TCM researchers have conducted substantial researches on the aetiology/pathogenesis and clinical treatment of SAP and have accumulated certain experiences. ${ }^{4}$ As some studies have shown, 'qi deficiency and blood stasis' and 'qi stagnation and blood stasis' are the two most common TCM syndromes of SAP ${ }^{67}$ Chinese patent medicines can improve the clinical symptoms of SAP patients, reduce the number of attacks, increase blood supply to coronary ar teries, improve myocardial ischaemia, and resist oxidation and thrombus formation.

At present, more than $70 \%$ of SAP patients in China are using Chinese patent medicines, ${ }^{9}$ and responding well to the treatment. Due to the lack of direct comparative effectiveness evidence about similar Chinese patent medicines, it is difficult for doctors to choose the optimal Chinese patent medicine for each patient. Needless to say, this increases the rate of irrational use and adverse events for Chinese patent medicines.

\section{Rational use of TCM}

'Syndrome differentiation and treatment' is the core of TCM theory. ${ }^{10} \mathrm{TCM}$ practitioners will summarise the major complaints of patients, differentiate their syndrome types 
based on an overall consideration and then prescribe medicines for them. Chinese patent medicines are the modern TCM medicine in different dosage forms, processed from different herbs under the guidance of TCM theories. However, according to investigations, $98 \%$ of users of Chinese patent medicines are persons ignorant of TCM theory and practice in China, giving rise to irrational use of these medicines and consequently limited efficacy. ${ }^{11}$ Thus, it is very important to identify and explain the efficacy of similar Chinese patent medicines in a simpler and clearer method.

\section{Identifying characteristics of Chinese patent medicines}

At the end of the 1990s, the concept of 'personalised medicine' was proposed and applied to the field of tutor treatment, representing the trend of medical development. The core of 'syndrome differentiation and treatment' of TCM is personalised diagnosis and treatment; identifying characteristics of Chinese patent medicines will help screen out the most effective medicine for individual patient.

COME-PIO (Comparative Effectiveness Research for similar Chinese patent medicines based on Patient Important Outcomes), built in the early stage by our research team, is a method for finding the characteristics of Chinese patent medicines. ${ }^{12}$ This method breaks the TCM syndrome down into a multiple of symptom combinations, then makes a comparison at the level of symptom or symptom combinations, and finally gives an individuality analysis based on the consolidated results among the comparison of different medicines and syndromes. This method now has integrated advanced analytical technologies, such as comparative effectiveness research (CER), ${ }^{13} 14$ patient important outcome (PIO),${ }^{15}$ patient report outcome (PRO), ${ }^{16}{ }^{17}$ minimal clinically important differences (MCID) $)^{18} 19$ and correspondence analysis $(\mathrm{CA}),{ }^{20}$ and is adopted in this study.
Two common Chinese patent medicines for SAP

Qishenyiqi Dripping Pills (QSYQ) and Compound Danshen Dripping Pills (FFDS) are two common Chinese patent medicines for treating SAP. The main ingredients of QSYQ are astragalus, salvia miltiorrhiza, pseudo-ginseng and rosewood heart wood; and the main ingredients of FFDS are salvia miltiorrhiza, pseudoginseng and borneol. The two medicines are in the same dosage form.

\section{Objective of this study}

This study will explain and differentiate the efficacy of QSYQ and FFDS from the perspective of improvement in patients' symptoms or symptom combinations, so as to promote rational use of them in clinical practice. The CUPID-based clinical trial model for personality identification of similar Chinese patent medicines will be designed and built in this study.

\section{METHODS}

\section{Research type}

This is a randomised controlled, double-blind and double-dummy, partial crossover design.

\section{Intervention}

This study comprises three stages (figure 1):

- First treatment (14 days): randomised controlled trial design is adopted

Group 1: Routine medication + A (QSYQ + FFDS placebo)

Group 2: Routine medication + B (FFDS + QSYQ placebo)

- Wash-out phase (3 days): all subjects enter the wash-out period, during which the first efficacy analysis is given

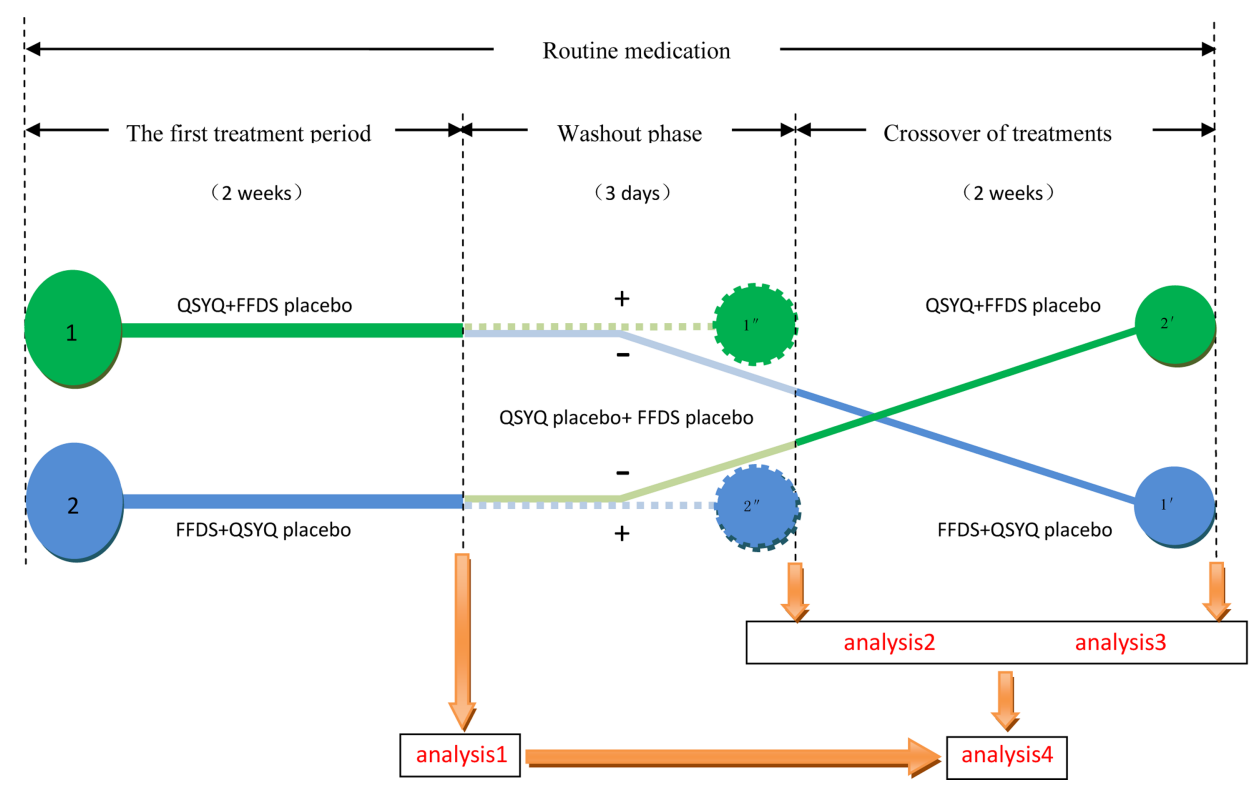

Figure 1 Flow chart of a CUPID method-based clinical trial design. 
Group 1: Routine medication + QSYQ placebo + FFDS placebo

Group 2: Routine medication + FFDS placebo + QSYQ placebo

- Crossover of treatment (14 days): subjects for whom the medicine is found ineffective in the first efficacy analysis will enter the crossover trial for further observation.

Group 1': Routine medication + B (FFDS + QSYQ placebo)

Group 2': Routine medication + A (QSYQ + FFDS placebo).

\section{Routine medication}

Routine medications include aspirin, angiotensinconverting enzyme inhibitors or angiotensin receptor blockers, $\beta$-receptor blockers, statins, nitrates and drugs for improving myocardial metabolism. In addition, participants cannot use the banned drugs listed in table 1 during the treatment period.

\section{Randomisation}

Patients are assigned randomly by the stratified blocked randomisation method (1:1); the stratification factor is syndrome pattern and symptom combination. The syndrome criteria of 'qi deficiency and blood stasis' and 'qi stagnation and blood stasis' are based on the basic components of the symptom combination; each combination comprises primary symptoms and secondary symptoms (primary symptoms are fixed, secondary symptoms are decided by the patients themselves). A third party statistician works out the Random Assignment Table using SAS V.9.1 (table 2).

\section{Allocation concealment}

A random number table generated by simulation of SAS statistical software is used for allocation concealment. Original copies of the blind codes are sealed in the lightproof envelope; one is kept by the major research unit and the other by the applicant of the trial. They are not allowed to be opened before formal statistical analysis. Drug blinding is carried out by the randomised group made up of members not involved in this trial; and the whole process is given strict supervision and quality control.

\section{Blindness}

This trial adopts the double-blind method. The trial drug and simulator for use are both provided by the manufacturer; they are basically identical in appearance, shape, colour and packaging, and are accompanied by a qualified drug inspection report. The principal investigators, clinical research assistants, drug administrators, patients and statisticians will be blinded.

In case of emergencies or necessary rescue of patients, persons-in-charge of the participating units shall immediately report to the clinical research associate and major investigators; unblinding can be performed only upon their approval. Once the allocation is unblinded,

Table 1 List of banned Chinese patent medicines

\begin{tabular}{|c|c|}
\hline 1. Salvia miltorrhiza tablets (丹参片) & 23. Huanxin Dan (环心丹) \\
\hline 2. Compound danshen tablets (复方丹参片) & 24. Diaoxinxue Kang (地奥心血康) \\
\hline 3. Compound salvia miltorrhiza injection (复方丹参注射液) & 25. Compound angelica injection (复方当归注射液) \\
\hline 4. Notoginseng total glucoside pieces (Electuary) (三七总式片(冲服剂)) & 26. Huoxin Dan (活心丹) \\
\hline $\begin{array}{l}\text { 5. Shuxuening tablets (Gin Kgo Plus, Taponin, Hua Baotong) (舒血宁片 } \\
\text { (银可络, 天保宁, 华宝通)) }\end{array}$ & 27. Guanxin pill (冠心片) \\
\hline 6. Xinkeshu tablets (心可舒片) & 28. KangShuan BaoXin tablets (抗栓保心片) \\
\hline 7. Guanxin Danshen tablets (冠心丹参片) & 29. Yixin pills (益心丸) \\
\hline 8. Shuguan tablets (舒冠片) & 30. Jiuxin oil (救心油) \\
\hline inkening (一洲心可宁) & 31. Maiguanyan tablets (脉管炎片) \\
\hline sules (康尔心胶囊) & 32. Guanmaining (冠脉宁) \\
\hline al liquid (心通口服液) & 33. Nuodikang capsule (诺迪康胶囊) \\
\hline on (血栓通注射液) & 34. Instant-effect Jiuxin pills (速效救心丸) \\
\hline 活血通脉胶囊) & 35. Xuefu Zhuyu capsule (血府逐瘀胶囊) \\
\hline 14. Guanxin Suhe pills (冠心苏合丸) & $\begin{array}{l}\text { 36. Haiwang Ginkgo biliba tablets (capsule) (海王 } \\
\text { 银杏叶片(胶囊)) }\end{array}$ \\
\hline Nakai)Kupr (pill) (活血丹( & 37. Yufengningxin tablets (愈风宁心片) \\
\hline 16. $\mathrm{Xi}$ & 38. Danqi tablets (丹七片) \\
\hline 17. Qiangli Naoxinkang (强力脑心康) & 39. Danshen Shuxin tablets (丹参舒心片) \\
\hline 18. Naoxintong (脑心通) & 40. Xinkeshu (心可舒) \\
\hline 19. Acanthopanax senticosus injection (刺五l注射液) & $\begin{array}{l}\text { 41. American golden ginkgo soft capsule (美国金 } \\
\text { 银杏软胶囊) }\end{array}$ \\
\hline 20. Xiaoshuan Zaizao pills (消栓再造丸) & 42. Diao Xinxuekang (地奥心血康) \\
\hline 21. Xinling pill (心灵丸) & 43. Shexiang Baoxin pill (㮋香保心丸) \\
\hline 22. Dantian Jiangzhi pills (丹田降脂丸) & 44. Xueshuan Xinmaining (血栓心脉宁) \\
\hline
\end{tabular}


Table 2 The two syndrome types and six symptom combination groups involved in this trial

\section{Number of symptom combination}

Combination 1

Combination 2

Combination 3

Combination 4

Combination 5

Combination 6
Type of symptom combination

+shortness of breath

+fatigue

+palpitations

+spontaneous qi deficiency and blood stasis

+perspiration syndrome

$\begin{array}{ll}\text { Chest pain+ chest } & \text { +spontaneous } \\ \text { tightness } & \text { +perspiration } \\ & \text { +chest coerces bloated }\end{array}$

+ pain

+palpitations
Syndrome differentiation

qi stagnation and blood stasis

syndrome the operation and record-taking must observe the requirements of the trial.

\section{Sample size}

The sample size was calculated on the basis of literature research. It was assumed that the means of the Seattle angina questionnaire (SAQ) scores of the groups taking QSYQ and taking FFDS were 82 and 80, respectively, with the same $\mathrm{SD}(\mathrm{SD}=4)$.

A total of 144 patients, 72 in each group, should be recruited in order to show a significant difference between the two groups, with a significance level of $0.05,80 \%$ power and a drop-out rate of $15 \%$ using the PASS software.

\section{Screening of participants}

- Screening condition

Patients participation in this trial is voluntary and they should select the symptom types by themselves; physicians should note the criteria met and diagnose syndrome types.

- Diagnosis criteria

SAP (I-III): diagnostic criteria refer to WHO for nomenclature and criteria for diagnosis of ischaemic heart disease and the Canadian Cardiovascular Society classification standard in $1972 .^{21}$

'Qi and blood stasis' and 'qi deficiency and blood stasis' syndrome: syndrome differentiation criteria refer to the Guidelines for Clinical Research of Chinese Medicine (new drug) in 2002. ${ }^{22}$

- Inclusion criteria

Patients aged between 40 and 75 .

Patients have signed informed consent forms.

Patients diagnosed with SAP.

Patients with SAP of grade I, II or III.

Patients with 'qi deficiency and blood stasis' or 'qi stagnation and blood stasis' syndromes.

- Exclusion criteria

Patients younger than 40 or older than 75 years.

Patients do not conform to diagnostic standards of Western medicine; TCM pattern is diagnostic.

Patients with infraction angina or Prinzmetal variant angina.

Patients with other organ dysfunction and other diseases involving the heart.
Patients with uncontrolled hypertension (systolic blood pressure $\geq 180 \mathrm{~mm} \mathrm{Hg}$ and/or diastolic blood pressure $\geq 110 \mathrm{~mm} \mathrm{Hg}$ ).

Patients who have received percutaneous coronary intervention for no more than 3 months.

Patients with a cardiac pacemaker.

Patients with a history of allergy to the control drug or investigational drug.

Patients with liver and kidney dysfunction.

Patients with tumours, autoimmune disease or blood disease, or pregnant or lactating women who should not be included in the trial as adjudged by the recruiting personnel.

Presence of active peptic ulcers and other haemorrhagic disease.

Patients involved in another clinical trial now or in the past 3 months.

- Termination criteria

Patients withdraw of their own accord for any reason.

Serious adverse events occurring during the trial.

Major mistakes or serious deviations identified in the clinical trial protocol in the process of execution (though the plan is good), making it difficult to evaluate the efficacy of the drug.

Trial is cancelled by the authority.

\section{Study setting}

We will prepare to collect cases from the first hospital and Baokang Hospital of Tianjin University of TCM, Tianjin Nankai Hospital and Wuqing hospital of TCM in China.

\section{Registration and enrolment}

Registration of patients

Two months prior to the trial, 144 SAP subjects qualified for inclusion will be gathered from four hospitals of Tianjin City (72 cases of 'qi stagnation and blood stasis', 72 cases of 'qi deficiency and blood stasis'); basic data of the patients, including name, sex, age, type of syndrome and contact, will be registered.

\section{Centralised enrolment}

The patients will be enrolled into groups altogether on the same day when the trial has commenced. Inclusion 
conditions of patients will be confirmed again based on inclusion criteria. Patients meeting inclusion conditions are randomised into one of two groups based on their syndrome and symptom types.

\section{Index of end point}

Score of SAQ: 19 questions in all, including physical limitation, anginal stability, anginal frequency, treatment satisfaction, and understanding of illness. The higher the score, the better the life quality and functional status of the organism will be. ${ }^{23}$

Score of Likert scale (LS): this scale contains a series of statements that expresses the positive and negative attitudes towards the test items and asks the respondents to express their degree of satisfaction. The answer of each respondent will be awarded certain points to show his or her degree of approval or disapproval of each statement. ${ }^{24}$

\section{Follow-up}

A total of four follow-up points are arranged in this trial: the first visit is day 0 after enrolment; the second visit is day $14 \pm 1$; the third visit is day $17 \pm 1$, and the fourth visit is day $31 \pm 1$.

Data are captured based on the CRF (table 3).

\section{Measurement tools (MCID) ${ }^{25}$}

Extract the SAQ values of Likert scale 7 of all patients in the interval of $(0,+1)$, the calculated mean value is marked as $\bar{x} 0$; extract the SAQ value of Likert scale 7 in the interval of $(+2,+3)$, the calculated mean value is marked as $\bar{x} 1$ (figure 2).
Calculation formula for MCID:

$$
\operatorname{MCID}=\sqrt{\frac{\sum(\mathrm{x} 1-\overline{\mathrm{x}} 1)^{2}+\sum(\mathrm{x} 0-\overline{\mathrm{x}} 0)^{2}}{(\mathrm{n}-1)}} \times(1-\mathrm{r})
$$

( $\mathrm{N}$ represents the sample size; $r$ represents the reliability coefficient of SAQ scale).

Compare SAQ value of each patient against MICD, which is regarded as the valid measurement scale. Results of comparisons show the efficacy on relevant symptoms or symptom combinations; values above MCID indicate effectiveness, values below MCID indicate ineffectiveness.

\section{STATISTICAL ANALYSIS Baseline balance}

Baseline demographic characteristics will be reported as mean and SD for continuous data and number/percentage for categorical data. Intergroup comparability is crucial to options of statistical methods. In this study, comparability will be checked by t test or $\chi^{2}$ test where appropriate. In case of incomparability, baseline-adjusted methods will be used.

\section{First treatment period}

After the first treatment period, the SAQ scores will be compared between the two groups using the test or Mann-Whitney $U$ test according to the normality of the sample distribution indicated by the KolmogorovSmirnov test. A two-tailed value of $\mathrm{p}<0.05$ will be considered statistically significant. Moreover, all patients will be

\begin{tabular}{|c|c|c|c|c|}
\hline \multirow[b]{2}{*}{ Items } & \multicolumn{4}{|l|}{ Visit } \\
\hline & $\begin{array}{l}1 \\
0 \text { day }\end{array}$ & $\begin{array}{l}2 \\
14 \pm 1 \text { days }\end{array}$ & $\begin{array}{l}3 \\
17 \pm 1 \text { days }\end{array}$ & $\begin{array}{l}4 \\
31 \pm 1 \text { days } \\
\end{array}$ \\
\hline $\begin{array}{l}\text { Medical history } \\
\text { Inclusion/exclusion criteria } \\
\text { Informed consent form } \\
\text { Syndrome differentiation } \\
\text { Symptom combination } \\
\text { Random allocation } \\
\text { General information } \\
\text { History of medical treatment and allergies } \\
\text { Taking drugs currently } \\
\text { Drug distribution } \\
\text { Drug recovery } \\
\text { Combination drugs } \\
\text { Compliance judgement }\end{array}$ & $\begin{array}{l}\sqrt{ } \\
\sqrt{ } \\
\sqrt{ } \\
\sqrt{ } \\
\sqrt{ } \\
\sqrt[V]{ } \\
\sqrt{ } \\
\sqrt[V]{ } \\
\sqrt[V]{ }\end{array}$ & $\begin{array}{l}\sqrt{ } \\
\sqrt{ } \\
\sqrt{ } \\
\sqrt{ }\end{array}$ & $\begin{array}{l}\sqrt{ } \\
\sqrt{ } \\
\sqrt{ }\end{array}$ & $\begin{array}{l}\sqrt{ } \\
\sqrt{ }\end{array}$ \\
\hline $\begin{array}{l}\text { Evaluation index } \\
\text { SAQ score } \\
\text { 7-point LS score }\end{array}$ & & $\sqrt{ }$ & & $\sqrt{ }$ \\
\hline $\begin{array}{l}\text { Safety observation } \\
\text { Vital signs } \\
\text { Adverse event } \\
\end{array}$ & $\sqrt{ }$ & $\sqrt{ }$ & $\sqrt{ }$ & $\sqrt{V}$ \\
\hline
\end{tabular}


Figure 2 Measurement of minimal clinically important differences (MCID).

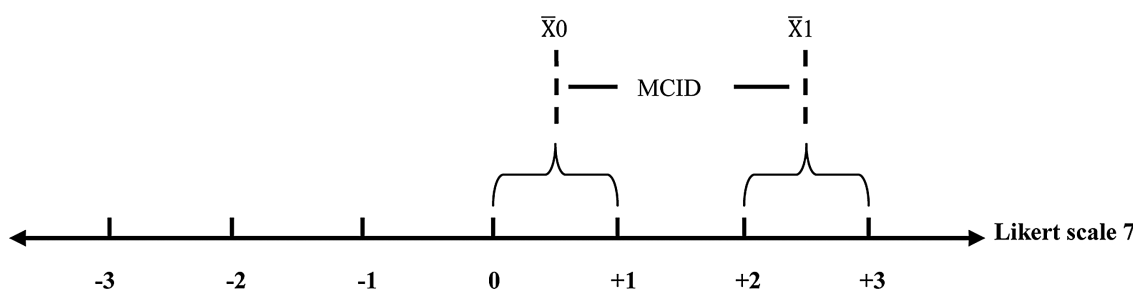

divided into different subgroups by single symptom combination or multiple symptom combinations. The SAQ scores will be compared between the two groups, in order to differentiate the characteristics of the two medicines.

\section{After the crossover treatment period}

The individual results of the first treatment period and the crossover treatment period are compared; CA is adopted to compare the curative effects of $\mathrm{A}$ and $\mathrm{B}$ on symptoms, symptom combinations and SAP-related TCM syndrome.

\section{Curative effect of treatment $A(B)$ on single symptom combination}

Establish corresponding relationship between symptom 1-6 and two outcomes: A is effective and B is ineffective (B is effective and A is ineffective); both $\mathrm{A}$ and $\mathrm{B}$ are ineffective.

\section{Curative effect of treatment $A(B)$ on symptom combinations}

List all possible multiple symptom combinations and establish corresponding relationship between different symptom combinations and the two outcomes: $\mathrm{A}$ is effective and B is ineffective (B is effective and A is ineffective); both $\mathrm{A}$ and $\mathrm{B}$ are ineffective.

\section{Curative effect of treatment $A(B)$ on TCM syndrome}

Merge the symptom according to different TCM syndromes and establish corresponding relationship between different symptom combinations and the two outcomes: A is effective and B is ineffective (B is effective and $\mathrm{A}$ is ineffective); both $\mathrm{A}$ and $\mathrm{B}$ are ineffective.

\section{Plot the corresponding distribution (Biplot)}

In this study, SPSS V.16.0 will be used to perform CA. The relative distances between different points will be calculated with the Biplot method; these will be the differences between treatment $\mathrm{A}$ and treatment $\mathrm{B}$.

\section{SAFETY}

\section{Standard operating procedures of adverse events}

Standard operating procedures (SOPs) for the management of adverse events (AEs) must be worked out in order to guarantee that AEs are under control. Clinical research associates (CRAs) will participate in AE management and SOP drafting so that they can manage AEs during clinical testing in a scientific and standardised manner.

\section{Recording of AEs}

When observing efficacy, pay attention to the occurrence of AEs and adverse reactions and record them in detail; serious AEs arising out of the trial must be reported in good time to the person-in-charge of the project and the ethics committee.

\section{Rating of AE severity}

The correlation between $\mathrm{AE}$ and drug is estimated according to 5-grade criteria (tables 4 and 5).

\section{Analysis of AEs}

The $\chi^{2}$ test is used to compare the incidence of AEs of drug $\mathrm{A}$ and $\mathrm{B}$, and the correlation between $\mathrm{AE}$ and drug is analysed.

\section{Drug management}

We will establish a trial drug management and register system. Trial drug management personnel must have passed good clinical practice (GCP) training and obtained a qualification certificate; they must possess the capability of managing clinical trial drugs. A central drug administrator takes charge of the overall allocation of all trial drugs; drug administrators of sub-centres take charge of the allocation and recovery of the drugs of their own centres. The whole process of acceptance, storage, allocation, use, counting and recovery of the trial drug are monitored by the CRA, Ethics Committee and Quality Control Committee, so as to ensure the safety and stability of the trial drugs.

\begin{tabular}{ll} 
Table 4 & Severity grading and definition \\
\hline $\begin{array}{l}\text { Severity } \\
\text { Grading }\end{array}$ & Definition \\
\hline Mild & $\begin{array}{l}\text { Short-lasting and mild symptoms, no pain } \\
\text { caused to patients, bearable, daily } \\
\text { activities not affected } \\
\text { Overt symptoms but bearable, daily } \\
\text { Moderate }\end{array}$ \\
Severe & $\begin{array}{l}\text { Severe symptoms, daily activities } \\
\text { seriously affected }\end{array}$ \\
\hline
\end{tabular}


Table 5 Determination of correlation between adverse event and drug

\begin{tabular}{llllll}
\hline Criteria & $\begin{array}{l}\text { Definitely } \\
\text { relevant }\end{array}$ & $\begin{array}{l}\text { Probably } \\
\text { relevant }\end{array}$ & $\begin{array}{l}\text { Probably } \\
\text { irrelevant }\end{array}$ & $\begin{array}{l}\text { Definitely } \\
\text { irrelevant }\end{array}$ & $\begin{array}{l}\text { Unable to } \\
\text { decide }\end{array}$ \\
\hline Within the reasonable post-dosage time sequence & + & + & - & - & - \\
Within known types of reaction of suspected drug & + & + & - & - & $?$ \\
Symptoms improved after withdrawal of drug & + & $?$ & $?$ & - & $?$ \\
Reactions recur after repeated administration & + & $?$ & $?$ & - & $?$ \\
Related to other treatment & - & + & + & & + \\
\hline '+' means YES; '-' means NO; '?' means unclear situation. & & &
\end{tabular}

\section{DATA MANAGEMENT}

\section{Management software}

This trial plans to use Oracle Clinical (OC) software for online data updating, data tracing and dynamic management at the same time, with the support of the check function of this software. ${ }^{26}$

\section{Data recording}

All data of the trial are subject to remote recording. Investigators will enter relevant data via the internet; such a pattern contributes to improved quality and efficiency of the clinical study.

\section{Data examination}

The data administrator performs a logic check and automatic comparison of data information using the check function of OC software, checks the result values are inconsistent with the case report forms, and checks one-by-one with the original case report forms and make corrections, so as to ensure the data in the database are consistent with the results of the case report form. This enables traceability, accuracy, completeness and timeliness of data.

\section{Data exporting}

After the trial, the data administrator will export the data in the form of data interexchange code and statistical analysts will extract relevant data from the database according to the code and program for statistical analysis.

\section{QUALITY ASSURANCE}

\section{Compliance of investigators}

Before the trial, all investigators must be trained as per the trial and technical requirements. The prime investigator is responsible for examining the case inclusion criteria of their units, deciding the end point and adverse events, handling serious AEs, controlling the trial quality of their own units, and confirming the completion of trial. $^{27}$

\section{Compliance of subjects}

Subjects will receive trial drugs, transportation fees and necessary healthcare instructions (diet, mental adjustment) for free. Subjects are required to maintain appropriate physical activities and control daily exercises, in order to guarantee inter-group comparability. The dosage and remaining amount of drug shall be recorded; the drug counting method is used to monitor compliance.

\section{Monitoring}

An Independent Data Monitor Committee (IDMC) composed of clinical experts, statisticians and relevant workers will provide regular monitoring of this trial. CRAs are required to monitor various units regularly; CRAs shall rigidly examine case report forms to ensure consistency with the original data, and they shall trace the source or directly visit the subjects when necessary; CRAs shall identify problems timely and feed back the solution to investigators within the shortest time.

\section{DISCUSSION}

Chinese patent medicines have definite advantages in treating SAP, particularly in improving symptoms of patients. In the past, many SAP patients have expressed their great satisfaction with Chinese patent medicines. Use of Chinese patent medicines is one of the major clinical practices based on 'syndrome differentiation and treatment' under the guidance of TCM. However, due to improper use by many non-TCM persons, irrational clinical use of Chinese patent medicines is frequently reported, which has seriously affected their clinical efficacy.

The CUPID-based clinical trial model built in this trial for identification of individual characteristics of similar Chinese patent medicines explains and distinguishes the efficacy of QSYQ and FFDS from a more intuitive angle of patients' symptoms or symptom combination, thus contributing to wider and more definite and rational use of Chinese patent medicines; this method provide direct evidence of the efficacy of these medicines by asking patients to choose their symptom types and evaluate the efficacy on their symptoms. A partial crossover trial design is used for classification evaluation of drugs and symptoms; the COME-PIO method is used to achieve 'reduced dimension decomposition, multidimensional comparison and degree progress analysis' of the TCM syndrome, enabling the expression of the efficacy of Chinese patent medicines in a more comprehensible way.

The CUPID model embodies advanced analysis technologies such as PIO, PRO, MCID and CA, and will provide a methodological reference for identifying the 
characteristic of Chinese patent medicines. Meanwhile, it will facilitate differentiated use of Chinese patent medicines by non-physicians and improve the use efficiency of Chinese patent medicines.

Acknowledgements This work was supported by the National Natural Science Foundation of China (grant 81202849) and Tianjin Higher education institution 'Innovative Team Training Program (N0.TD12-5032)'. We especially wish to acknowledge the cooperation of the research staff.

Contributors All authors have contributed to the overall design of this study, and been involved in the ongoing management of the trial. HBC plotted the study, participated in its design and coordination, prepared the protocol, wrote the manuscript, and undertook the staff management. NL participated in the design and coordination of the study, and wrote the manuscript. JBZ was in charge of sample size and all statistical works of trial. HXC, XL and WM collected and analysed the data and critically revised the manuscript. HCS conceived of and designed the study, obtained financial support and wrote the manuscript. ZL and HW were responsible for data management and developing, overseeing the qualitative components of the trial. All authors have read and approved the final manuscript.

Competing interests None.

Ethics approval The medical ethics committee of Tianjin University of TCM

Provenance and peer review Not commissioned; internally peer reviewed.

Open Access This is an Open Access article distributed in accordance with the Creative Commons Attribution Non Commercial (CC BY-NC 4.0) license, which permits others to distribute, remix, adapt, build upon this work noncommercially, and license their derivative works on different terms, provided the original work is properly cited and the use is non-commercial. See: http:// creativecommons.org/licenses/by-nc/4.0/

\section{REFERENCES}

1. Forood A, Malekpour-Afshar R, Sarnevesht J. Effect of opium addiction on aspirin resistance in stable angina pectoris. Addict Health 2014;6:7-13.

2. Fox K, Garcia MA, Ardissino D, et al. Guidelines on the management of stable angina pectoris: executive summary: The Task Force on the Management of Stable Angina Pectoris of the European Society of Cardiology. Eur Heart J 2006;27:1341-81.

3. Jamal S, Scaria V.; Open Source Drug Discovery Consortium. Data-mining of potential antitubercular activities from molecular ingredients of traditional Chinese medicines. Peer J 2014;2:e476.

4. Food and Drug Administration: Guidance for Industry on Complementary and Alternative Medicine Products and Their Regulation. 2006. http:// www.fda.gov/downloads/Regulatorylnformation/Guidances/ UCM145405.pdf

5. Mohammed A, David FS, Amadou KS. Safety and efficacy of ranolazine for the treatment of chronic angina pectoris. Clin Med Insights Ther 2013:5:1-14.

6. Qiu Y, Xu H, Shi DZ. Traditional chinese herbal products for coronary heart disease: an overview of cochrane reviews. Evid Based Complement Alternat Med 2012. http://www.ncbi.nlm.nih.gov/ pmc/articles/PMC3320001/pdf/ECAM2012-417387.pdf
7. The people's Republic of China State Administration of traditional Chinese Medicine: Standard of diagnosis and therapeutic effect for diseases and syndromes in traditional Chinese Medicine. Nanjing: Nanjing University Press, 1994:18.

8. Wang G, Wang L, Xiong ZY, et al. Compound salvia pellet, a traditional Chinese medicine, for the treatment of chronic stable angina pectoris compared with nitrates: a meta-analysis. Med Sci Monit 2006;12:SR1-7.

9. Wei M. Choose of Chinese patent medicine for the treatment of coronary heart disease. Chin J Intergr Med Cardio-/Cerebrovasc Dis 2008;6:89-90

10. Tang JL, Liu BY, Ma KW. Traditional Chinese medicine. Lancet 2008;372:1938-40.

11. Wang $Y G$, Jin $P$, Chen $H$. Analysize the problems existing in the Use of Chinese Patent Medicine. Chin J Geriatric Care 2012;10:114-15.

12. Cao HB, Zhai JB, Mu W, et al. Use of comparative effectiveness research for similar Chinese patent medicine for angina pectoris of coronary heart disease: a new approach based on patient-important outcomes. Trials 2014;15:84.

13. NIH: Comparative Effectiveness Research (CER). 2010. http://www. nlm.nih.gov/hsrinfo/cer.html

14. Johnson SP, Chung KC. Comparative effectiveness research in hand surgery. Hand Clin 2014;30:319-27.

15. Hoang-Kim A, Miclau T, Goldhahn J, et al. Patient-important outcome for the assessment of fracture repair. Injury 2014;(Suppl 2):S44-8

16. Valdes $\mathrm{K}$, MacDermid J, Algar $\mathrm{L}$, et al. Hand therapist use of patient report outcome (PRO) in practice: a survey study. J Hand Ther 2014;:27:299-308.

17. Johnston BC, Patrick DL, Thorlund K, et al. Patient-reported outcomes in meta-analyses-part 2: methods for improving interpretability for decision-makers. BMC Health Qual Life Outcomes 2013;11:211.

18. Chan LS. Minimal clinically important difference (MCID)-adding meaning to statistical inference. Am J Public Health 2013;103:e24-5.

19. Binenbaum Y, Amit M, Billan S, et al. Minimal clinically important differences in quality of life scores of oral cavity and oropharynx cancer patients. Ann Surg Oncol 2014;21:2773-81.

20. Infantosi AF, Costa JC, Almeida RM. Correspondence analysis:a theoretical basis for categorical data interpretation in health sciences. Cad Saude Publica 2014;30:473-86.

21. [No authors listed]. Dignosis and treatment of chronic stable angina. Chin J Cardiol 2007;35:195-206.

22. Zheng XY. Clinical guideline of new drugs for traditional Chinese medicine. Beijing: The Medicine Science and Technology Press of China, 2002;69.

23. Spertus JA, Winder JA, Dewhurst TA, et al. Development and evaluation of the Seattle Angina Questionnaire: a new functional status measure for coronary artery disease. J AM Coll Cardiol 1995:25:333-41.

24. Weber CK, Miglioranza MH, de Moraes MAP, et al. The five-point Likert scale for dyspnea can properly assess the degree of pulmonary congestion and predict adverse events in heart failure outpatients. Clinics 2014;69:341-6.

25. Landorf KB, Radford JA, Hudson S. Minimal Important Difference (MID) of two commonly used outcome measures for foot problems. $J$ Foot Ankle Res 2010;10:7-12.

26. Oracle: Powering Clinical Studies with Oracle $2010 \mathrm{http}: / / \mathrm{www}$. oracle.com/us/industries/life-sciences/045788.pdf

27. Cao HB, Shang HC, Mu W, et al. Internal challenge to clinical trial project management: strategies for managing investigator compliance. J Evid Based Med 2013;6:157-65. 